\title{
Nichts ist wirklich sicher
}

\author{
WISSENSCHAFT ERKLÄRT: WAHRSCHEINLICHKEITEN Auf die Frage, ob bestimmte \\ Ereignisse eintreffen werden, kann man häufig nur mit „wahrscheinlich schon“ \\ oder „wahrscheinlich nicht" antworten. Für Physiotherapeuten ist es wichtig, Wahr- \\ scheinlichkeiten in ihre Entscheidungsfindung mit einzubeziehen - aber reflektiert.
}

D

ie Wahrscheinlichkeit ist eine Einstufung oder Graduierung der Sicherheit von bestimmten Aussagen. In Studien dienen viele Berechnungen dazu, herauszufinden, wie sicher bestimmte Ereignisse eintreffen. Wahrscheinlichkeiten tauchen dabei in verschiedenen Zusammenhängen auf.

Erkrankungsrisiko berechnen > Berechnen kann man beispielsweise das sogenannte Relative Risiko (RR), um die Wahrscheinlichkeit des Ausbruchs einer Krankheit herauszufinden: Das RR beschreibt den Effekt einer bestimmten Exposition auf eine Erkrankung. Durch seine Berechnung können Forscher zum Beispiel herausfinden, ob Raucher im Verhältnis zu Nichtrauchern mit höherer Wahrscheinlichkeit an Lungenkrebs erkranken werden. Dabei berechnen die Forscher das RR, indem sie den Quotienten aus Erkrankten (Lungenkrebs) und Exponierten (Rauchern) teilen durch den Quotienten aus Erkrankten und Nichtexponierten (Nichtrauchern).

$\mathbf{R R}=\frac{\frac{\text { Erkrankte }}{\text { Exponierte }}}{\frac{\text { Erkrankte }}{\text { Nichtexponierte }}}$

Ist das Ergebnis $>1$, dann ist das Risiko einer Erkrankung erhöht. Ein - in diesem Beispiel unrealistisches - RR von weniger als 1 würde dagegen bedeuten, dass Rauchen vor Lungenkrebs schützt, also präventiv wirkt.

Solche globalen Ergebnisse sind aber nicht zwangsläufig auf Einzelpersonen übertragbar.
Betrachten wir dazu „Altkanzler“ Helmut Schmidt: Obwohl es unbestritten so ist, dass Raucher oftmals früher sterben als Nichtraucher, feierte der bekennende Kettenraucher Schmidt gerade seinen 90. Geburtstag.

Therapieerfolg abschätzen > Für die physiotherapeutische Praxis ist es beispielsweise interessant, wenn Forscher die Effektivität verschiedener Therapieansätze bei bestimmten Krankheitsbildern untersuchen. Ein anschauliches Beispiel dafür bietet der Vergleich zwischen dem Umlagerungstraining nach Semont und dem nach Epley (॰ Abb.) in der Behandlung des sogenannten benignen paroxysmalen Lagerungsschwindels.

Dr. Andreas Radtke und seine Kollegen untersuchten in einer Studie, wie hoch die Wahrscheinlichkeit ist, dass man Patienten durch die Anwendung einer dieser Therapien von dem Schwindel befreien kann. Die Forscher fanden heraus, dass beide Behandlungsansätze effektiv sind. Allerdings konnten sie den Schwindel der Patienten durch die Anwendung des Epley-Manövers schneller reduzieren als durch die Umlagerung nach Semont. Durch die Behandlung nach Epley wurden zusätzlich mehr Probanden beschwerdefrei [1]. Die Schlussfolgerung, die man ziehen kann, ist, dass es wahrscheinlicher ist, betroffenen Patienten mit dem Manöver nach Epley helfen zu können. Das Lagerungstraining nach Semont dagegen wäre wahrscheinlich nicht so erfolgversprechend.
Ausnahmen nicht verpassen > Verlässt man sich blind darauf, dass eine bestimmte Therapie den Patienten mit hoher Wahrscheinlichkeit helfen wird, birgt dieses Vertrauen immer ein Risiko: die Ausnahmen zu verpassen. Selbst wenn ein Ergebnis höchstwahrscheinlich eintreten wird, ist das kein Garant dafür, dass tatsächlich ausnahmslos alle Patienten so reagieren werden - siehe Altkanzler Schmidt. Auch in unserem Beispiel bedeuten die Ergebnisse weder, dass der Therapieerfolg auf sämtliche Patienten mit paroxysmalem Lagerungsschwindel übertragbar ist, noch, dass die Therapie nach Epley immer effektiver ist als die nach Semont. Es gibt lediglich eine höhere Wahrscheinlichkeit, dass man mit dem EpleyManöver den Schwindel zuverlässiger therapieren kann. Aufgrund dieser nie ganz auszuschließenden Unsicherheit steht für Physiotherapeuten die individuelle Befundaufnahme weiterhin an erster Stelle, wenn sie ihre Patienten behandeln.

Das Wissen um den wahrscheinlich effektivsten Behandlungsansatz bei einem bestimmten Krankheitsbild ist dennoch unbestritten von Vorteil und sollte auf jeden Fall in die Entscheidung für den Behandlungsplan mit einfließen. Denn evidenzbasierte Praxis bedeutet, neben der eigenen Erfahrung auch Wahrscheinlichkeiten angemessen bei jedem Patienten zu berücksichtigen.

Prof. Dr. Jan Mehrholz

\section{$\Rightarrow$ Literatur: [1] Neurology 2004, 63; 8-9}
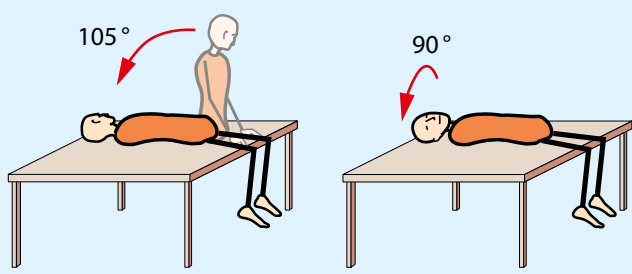

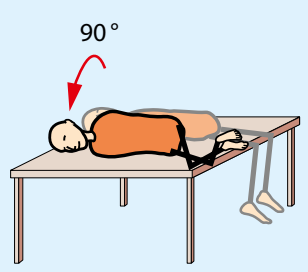

Abb. Beispiel für ein Manöver nach Epley bei linksseitigem Lagerungsschwindel. Die Wahrscheinlichkeit ist hoch, dass Betroffene dadurch beschwerdefrei werden. 